PROCEEDINGS OF THE

AMERICAN MATHEMATICAL SOCIETY

Volume 126, Number 6, June 1998, Pages 1835-1844

S 0002-9939(98)04425-6

\title{
A NORMAL SCREENABLE NONPARACOMPACT SPACE IN ZFC
}

\author{
ZOLTAN T. BALOGH
}

(Communicated by Franklin D. Tall)

\begin{abstract}
We construct a normal, screenable, nonparacompact space in ZFC. The existence of such a space is also known to imply that there is a normal, screenable space which is not collectionwise normal.
\end{abstract}

\section{INTRODUCTION}

Screenability (the property that every open cover has a $\sigma$-disjoint open refinement) was introduced and studied in R.H. Bing's 1951 paper on metrization [B]. An interesting question left open, first appearing four years later in a paper by K. Nagami $[\mathrm{N}]$, asks whether normality and screenability together are equivalent to paracompactness. The problem was restated as Classic Problem III in the problem section of the first volume of Topology Proceedings in 1976 ([TP], pp. 363-4). Seven years later, M.E. Rudin [R1] constructed a counter-example using $\diamond^{++}$, a combinatorial principle valid under the assumption of $V=L$.

The subject matter of this paper is to settle this problem in ZFC:

Theorem I. There is a normal, screenable space which is not paracompact.

Theorem I also answers Problem 51 in [W].

By another result of M.E. Rudin [R2], it follows from Theorem I that there is a normal, screenable space which is not collectionwise normal, answering a question of F. Tall [T1], [T2]. (Cf. Theorem 6.2.)

Sections 1-5 in our paper are devoted to the proof of Theorem I. Section 6 contains some concluding remarks.

Our terminology and notation follows the standards of set-theoretic topology $[\mathrm{KV}]$. Space means Hausdorff topological space. By a result of Nagami [N], to prove that a normal, screenable space is not paracompact, we have to show that it is not even countably paracompact. The following simple proposition follows from the standard characterization of countable paracompactness given by C.H. Dowker $[\mathrm{D}]$.

Proposition 0.1. Suppose that a space $X$ has an increasing open cover $\left\langle W_{n}\right\rangle_{n \in \omega}$ such that for every sequence $\left\langle G_{n}\right\rangle_{n \in \omega}$ of open sets with $G_{n} \supset X \backslash W_{n}(n \in \omega)$ we have $\bigcap_{n \in \omega} G_{n} \neq \emptyset$. Then $X$ is not countably paracompact.

Received by the editors March 5, 1996.

1991 Mathematics Subject Classification. Primary 54D15, 54D20; Secondary 54G20.

Key words and phrases. Normal, screenable, paracompact, collectionwise normal.

The author's research was partially supported by NSF Grant DMS-9623391.

(c)1998 American Mathematical Society 


\section{The Construction of $X$}

The underlying set of $X$ is $\mathfrak{c} \times \omega$, which we also denote by $X$. Let $\pi: X \rightarrow \mathfrak{c}$ be the natural projection. For every $n \in \omega$, let $L_{n}=\mathfrak{c} \times\{n\}$ and $W_{n}=\mathfrak{c} \times n\left(=\bigcup_{k<n} L_{k}\right)$. For every $x \in X$, let $B_{x}=X \backslash\{x\}$, and let

$$
\mathfrak{B}_{0}=\left\{B_{x}: x \in X\right\} \cup\left\{W_{n}: n \in \omega\right\} .
$$

$\mathfrak{B}_{0}$ will be part of a base for the topology of $X$ to be defined. Including the sets $B_{x}$ makes sure that $X$ is $T_{1}$, and $\left\{W_{n}\right\}_{n \in \omega}$ is an increasing open cover of $X$.

In order to make $X$ normal, we need the concept of a Type 1 pair. $S=\left\langle S^{0}, S^{1}\right\rangle$ is called a Type 1 pair iff $S^{0} \cup S^{1}=X$. Some of these Type 1 pairs will be open covers of the topology of $X$ (to be constructed), in which case we will give them a clopen disjoint refinement $\left\langle B^{0}, B^{1}\right\rangle$.

We are also going to make each subspace $W_{n}$ (ultra) paracompact, thereby making sure that $X$ is screenable. To do this, let us say that a sequence $\left\langle S^{\rho}\right\rangle_{\rho<\mathfrak{c}}$ is a Type 2 sequence of height $n$ iff $\bigcup_{\rho<c} S^{\rho}=W_{n}$. Some of these Type 2 sequences will be open covers of $W_{n}$, in which case we give them a clopen (in $W_{n}$ ) disjoint refinement $\left\langle E^{\rho}\right\rangle_{\rho<\mathfrak{c}}$.

Finally, to make sure that $X$ is not (countably) paracompact we will do the construction so that $\left\{W_{n}\right\}_{n \in \omega}$ becomes an open cover with no locally finite refinement. Control pairs defined below will help us achieve that.

For every subset $A$ of $X$, let

$$
S \uparrow A=\left\{\begin{array}{l}
\left\langle S^{0} \cap A, S^{1} \cap A\right\rangle \text { if } S \text { is a Type } 1 \text { sequence, } \\
\left\langle S^{\rho} \cap A\right\rangle_{\rho \in \pi(A)}, \quad \text { if } \mathrm{S} \text { is a Type } 2 \text { sequence. }
\end{array}\right.
$$

Definition 1.1. A pair $\langle A, d\rangle$ is called a control pair if the following conditions hold:

(C-1) $A$ is a countably infinite subset of $X$;

(C-2) $d$ is a countable function with $\operatorname{dom}(d) \subset\{S \uparrow A: S$ is a Type 1 or Type 2 sequence $\}$ and range $(d) \subset \mathfrak{c}$.

Let $\left\langle A_{\beta}, d_{\beta}\right\rangle_{\beta<\mathfrak{c}}$ list all control pairs, mentioning each $\mathfrak{c}$ times.

Let $\left\langle S_{\xi}\right\rangle_{\xi<2^{\mathrm{c}}}$ be a list of all Type 1 or Type 2 sequences, mentioning each $2^{\mathrm{c}}$ times. By transfinite induction on $\xi$ we are going to define an increasing sequence $\left\langle\mathfrak{B}_{\xi}\right\rangle_{\xi<2^{\text {c }}}$ of families of subsets of $X$. The topology of $X$ will be generated by $\mathfrak{B}=\bigcup_{\xi<2^{c}} \mathfrak{B}_{\xi}$ as a subbase. We will say that $Y$ is $\xi$-open ( $\xi$-closed) if $Y$ is open (closed) in the topology on $X$ generated by $\mathfrak{B}_{\xi}$ as a subbase.

$\mathfrak{B}_{0}$ was defined at the beginning of this section.

Suppose now that $0<\tau<2^{\mathfrak{c}}$ and we have already defined $\mathfrak{B}_{\xi}$ for every $\xi<\tau$.

If $\tau$ is a limit ordinal, then let $B_{\tau}=\bigcup_{\xi<\tau} B_{\xi}$.

If $\tau=\xi+1$, then we consider three cases, depending on $\xi$.

Case 1. Assume that $S_{\xi}=\left\langle S_{\xi}^{0}, S_{\xi}^{1}\right\rangle$ is a Type 1 pair, $S_{\xi}^{0}$ and $S_{\xi}^{1}$ are both $\xi$-open, and there is no $\eta<\xi$ such that $S_{\eta}=S_{\xi}$ and $S_{\eta}^{0}$, $S_{\eta}^{1}$ are both $\eta$-open.

In this case we are going to split $X$ into two disjoint subsets $B_{\xi}^{0}$ and $B_{\xi}^{1}$ such that $B_{\xi}^{i} \subset S_{\xi}^{i}$ for $i=0,1$. Then we set

$$
\mathfrak{B}_{\xi+1}=\mathfrak{B}_{\xi} \cup\left\{B_{\xi}^{0}, B_{\xi}^{1}\right\} .
$$

If $x=\langle\beta, k\rangle \in X$, then let us write $x(\xi)=i($ or $\langle\beta, k\rangle(\xi)=i)$ iff $x \in B_{\xi}^{i}$. 
To define $\left\langle B_{\xi}^{0}, B_{\xi}^{1}\right\rangle$ we have to decide, for each $\beta<\mathfrak{c}$, the values $\langle\beta, k\rangle(\xi), k \in \omega$. We consider two subcases, depending on $\beta$.

Subcase 1.a. Suppose that $S_{\xi} \uparrow A_{\beta} \in \operatorname{dom}\left(d_{\beta}\right)$ and $d_{\beta}\left(S_{\xi} \uparrow A_{\beta}\right) \in\{0,1\}$. Then, with the notation $d_{\beta}\left(S_{\xi}\left\lceil A_{\beta}\right)=i\right.$, let's set, for each $k \in \omega$,

$$
\langle\beta, k\rangle(\xi)= \begin{cases}i, & \text { if }\langle\beta, k\rangle \in S_{\xi}^{i} \\ 1-i, & \text { otherwise }\end{cases}
$$

Subcase 1.b. If Subcase 1.a does not hold, then set, for every $k \in \omega$,

$$
\langle\beta, k\rangle(\xi)= \begin{cases}0, & \text { if }\langle\beta, k\rangle \in S_{\xi}^{0} \\ 1, & \text { otherwise. }\end{cases}
$$

Case 2. Assume that $S_{\xi}=\left\langle S_{\xi}^{\rho}\right\rangle_{\rho<\mathrm{c}}$ is a Type 2 sequence of some height $n \in \omega, S_{\xi}^{\rho}$ is $\xi$-open for every $\rho<\mathfrak{c}$, and there is no $\eta<\xi$ such that $S_{\eta}=S_{\xi}$ and $S_{\eta}^{\rho}$ is $\eta$-open for every $\rho<\mathfrak{c}$.

In this case we are going to define a partition $\left\langle E_{\xi}^{\rho}\right\rangle_{\rho<\mathfrak{c}}$ of $W_{n}$ into pairwise disjoint sets such that $E_{\xi}^{\rho} \subset S_{\xi}^{\rho}$ for every $\rho<\mathfrak{c}$. (Some of the sets $S_{\xi}^{\rho}$ and $E_{\xi}^{\rho}$ may be empty.) Then we set

$$
\mathfrak{B}_{\xi+1}=\mathfrak{B}_{\xi} \cup\left\{E_{\xi}^{\rho}: \rho<\mathfrak{c}\right\} .
$$

For each $x=\langle\beta, k\rangle \in W_{n}$, let us write $x(\xi)=\rho($ or $\langle\beta, k\rangle(\xi)=\rho)$ iff $x \in E_{\xi}^{\rho}$. To define $\left\langle E_{\xi}^{\rho}\right\rangle_{\rho<\mathfrak{c}}$ we have to decide, for each $\beta<\mathfrak{c}$, the values $\langle\beta, k\rangle(\xi), k<n$. Depending on $\beta$, we consider two subcases.

Subcase 2.a. Suppose $S_{\xi}\left\lceil A_{\beta} \in \operatorname{dom}\left(d_{\beta}\right)\right.$. Then, with the notation $d_{\beta}\left(S_{\xi}\left\lceil A_{\beta}\right)=\right.$ $\rho$, let's set, for each $k<n$,

$$
\langle\beta, k\rangle(\xi)=\left\{\begin{array}{l}
\rho, \text { if }\langle\beta, k\rangle \in S_{\xi}^{\rho} ; \\
\min \left\{\sigma:\langle\beta, k\rangle \in S_{\xi}^{\sigma}\right\}, \text { otherwise. }
\end{array}\right.
$$

Subcase 2.b. If Subcase 2.a does not hold, then set $\langle\beta, k\rangle(\xi)=\min \left\{\sigma:\langle\beta, k\rangle \in S_{\xi}^{\sigma}\right\}$ for every $k<n$.

Case 3. If neither Case 1 nor Case 2 holds for $\xi$, then let $\mathfrak{B}_{\xi+1}=\mathfrak{B}_{\xi}$.

Having finished the construction of $\left\langle\mathfrak{B}_{\xi}\right\rangle_{\xi<2^{\mathfrak{c}}}$ (and thus, the construction of the topology of $X$ ), let us set

$$
\begin{aligned}
H_{1} & =\left\{\xi<2^{\mathfrak{c}}: \text { Case } 1 \text { holds for } \xi\right\}, \\
H_{2} & =\left\{\xi<2^{\mathfrak{c}}: \text { Case } 2 \text { holds for } \xi\right\}, \\
H & =H_{1} \cup H_{2} .
\end{aligned}
$$

Note that by the minimality of $\xi$ in Cases 1 and 2 we have the following.

Proposition 1.2. If $\xi, \eta \in H$ and $S_{\xi}=S_{\eta}$, then $\xi=\eta$.

Finally, by the definition of $\langle\beta, k\rangle(\xi)$ in Subcases 1.a and 2.a we have the following. 


\section{Observation 1.3.}

(a) If $\xi \in H_{1}$, then to prove $\langle\beta, k\rangle \in B_{\xi}^{i}$ it is enough to show that $\langle\beta, k\rangle \in S_{\xi}^{i}$ and $d_{\beta}\left(S_{\xi}\left\lceil A_{\beta}\right)=i\right.$.

(b) If $\xi \in H_{2}$, then to prove $\langle\beta, k\rangle \in E_{\xi}^{\rho}$ it is enough to show that $\langle\beta, k\rangle \in S_{\xi}^{\rho}$ and $d_{\beta}\left(S_{\xi}\left\lceil A_{\beta}\right)=\rho\right.$.

\section{2. $X$ IS NORMAL AND SCREENABLE}

Proposition 2.1. $X$ is normal.

Proof. It is enough to show that every open cover $\left\langle U_{0}, U_{1}\right\rangle$ of $X$ by two open sets has a shrinking $\left\langle B_{0}, B_{1}\right\rangle$ into disjoint clopen sets. Since $|X|=\mathfrak{c}, U_{0}$ and $U_{1}$ are both unions of $\leq \mathfrak{c}$ basic open sets. By $c f\left(2^{\mathfrak{c}}\right)>\mathfrak{c}$, there is a $\Theta<2^{\mathfrak{c}}$ such that $U_{0}$ and $U_{1}$ are both $\xi$-open for $\xi \geq \Theta$. Since each Type 1 pair is listed in $\left\langle S_{\xi}\right\rangle_{\xi<2^{\mathfrak{c}}} 2^{\mathrm{c}}$ many times, there is a $\xi<2^{\mathfrak{c}}$ such that $S_{\xi}=\left\langle U_{0}, U_{1}\right\rangle$ and $U_{0}$ and $U_{1}$ are $\xi$-open. Let us take the smallest such $\xi$. Then $\xi \in H_{1}$ and by Case 1 in the construction of $X,\left\langle B_{\xi}^{0}, B_{\xi}^{1}\right\rangle$ is a shrinking of $\left\langle U_{0}, U_{1}\right\rangle$ into disjoint clopen sets.

Remark. The above proof shows that $X$ is even strongly zero-dimensional.

Proposition 2.2. $X$ is screenable.

Proof. We are going to show that each of the open subspaces $W_{n}, n \in \omega$, is ultraparacompact, i.e. every open cover of $W_{n}$ has a refinement by pairwise disjoint clopen subspaces. To prove this, fix $n$, and let $\left\langle U^{\rho}\right\rangle_{\rho<\mathfrak{c}}$ be an open cover of $W_{n}$, with repetitions permitted. As in the proof of Proposition 2.1 it follows that there is a smallest $\xi<2^{\mathfrak{c}}$ such that $S_{\xi}=\left\langle U^{\rho}\right\rangle_{\rho<\mathfrak{c}}$, and $U^{\rho}$ is $\xi$-open for every $\rho<\mathfrak{c}$. Then $\xi \in H_{2}$, and by Case 2 in the construction of $X,\left\langle E_{\xi}^{\rho}\right\rangle_{\rho<\mathfrak{c}}$ is a refinement of $\left\langle U^{\rho}\right\rangle_{\rho<\mathfrak{c}}$ by pairwise disjoint clopen (in $W_{n}$ ) subsets of $W_{n}$.

The proof that $X$ is not (countably) paracompact will be contained in Sections 4 and 5 .

\section{Complete neighborhoods}

Let $x=\langle\beta, k\rangle \in X$. By the definition of the topology of $X$, the basic open neighborhoods of $x$ have the form

$$
V_{t, K}(x)=\bigcap_{\xi \in t} T_{\xi}(x) \cap\left(W_{k+1} \backslash K\right),
$$

where $t \in[H]^{<\omega}, K \in\left[W_{k+1}\right]^{<\omega}$, and for every $\xi \in t$,

$$
T_{\xi}(x)= \begin{cases}B_{\xi}^{x(\xi)}, & \text { if } \xi \in H_{1}, \\ E_{\xi}^{x(\xi)}, & \text { if } \xi \in H_{2} .\end{cases}
$$

For every $\xi<2^{\mathfrak{c}}$, let

$$
V_{t, K, \xi}(x)=\bigcap_{\eta \in t \cap \xi} T_{\eta}(x) \cap\left(W_{k+1} \backslash K\right) .
$$

Definition 3.1. A neighborhood $V_{t, K}(x)$ of $x \in X$ is said to be complete if for every $\xi \in t$,

$$
V_{t, K, \xi}(x) \subset S_{\xi}^{x(\xi)} .
$$

Otherwise, $V_{t, K}(x)$ is called incomplete. 
The following observation will be useful later.

Lemma 3.2. If $V_{t, K}(x)$ is a neighborhood of $x=\langle\beta, k\rangle \in X$, then there are $t^{*} \supset$ $t, K^{*} \supset K$ such that $V_{t^{*}, K^{*}}(x)$ is a complete neighborhood of $x$.

Proof. For every incomplete neighborhood $V_{t^{\prime}, K^{\prime}}(x)$ of $x$ with $t^{\prime} \supset t$ and $K^{\prime} \supset K$, let $\xi_{t^{\prime}, K^{\prime}}$ be the smallest $\xi \in t^{\prime}$ such that $V_{t^{\prime}, K^{\prime}, \xi}(x) \not \subset S_{\xi}^{x(\xi)}$. Our lemma then follows from the following claim by the fact that $2^{\mathfrak{c}}$ is well-founded.

Claim. If $t^{\prime} \supset t, K^{\prime} \supset K$ and $V_{t^{\prime}, K^{\prime}}(x)$ is an incomplete neighborhood of $x$, then there are $t^{\prime \prime} \supset t^{\prime}, K^{\prime \prime} \supset K^{\prime}$ such that $V_{t^{\prime \prime}, K^{\prime \prime}}(x)$ is either a complete neighborhood of $x$ or an incomplete neighborhood with $\xi_{t^{\prime \prime}, K^{\prime \prime}}<\xi_{t^{\prime}, K^{\prime}}$.

To prove the claim, let $\eta=\xi_{t^{\prime}, K^{\prime}}$. Since $S_{\eta}^{x(\eta)}$ is $\eta$-open, there are $\bar{t} \in[H \cap \eta]^{<\omega}$ and $\bar{K} \in[X]^{<\omega}$ such that $V_{\bar{t}, \bar{K}}(x)$ is a neighborhood of $x$ with $V_{\bar{t}, \bar{K}}(x) \subset S_{\eta}^{x(\eta)}$. Then $t^{\prime \prime}=t^{\prime} \cup \bar{t}, K^{\prime \prime}=K^{\prime} \cup \bar{K}$ are as required.

\section{4. $X$ is not COUNTABly PARACOMPACT: REFLECTING THE SETS $G_{k}$}

Let us arbitrarily choose a decreasing sequence of open sets $G_{k} \supset X \backslash W_{k}, k \in \omega$. By Lemma 0.1 , in order to show that $X$ is not countably paracompact, it is enough to show that $\bigcap_{k \in \omega} G_{k} \neq \emptyset$. This will take up Sections 4 and 5 , during which we keep $\left\langle G_{k}\right\rangle_{k \in \omega}$ fixed.

For every $k \in \omega$, let $\xi_{k}$ denote the unique element of $H_{1}$ such that $S_{\xi_{k}}=$ $\left\langle W_{k}, G_{k}\right\rangle$. Note that $B_{\xi_{k}}^{0} \subset W_{k}, B_{\xi_{k}}^{1} \subset G_{k}$ is a partition of $X$ into disjoint clopen sets. For every $x=\langle\beta, k\rangle \in X$, let $V(x)=V_{t(x), K(x)}(x)$ be a neighborhood of $x$ such that $\left\{\xi_{j}: j \leq k\right\} \subset t(x)$, and thus, $V(x) \subset B_{\xi_{k}}^{1} \subset G_{k}$. Let $t_{i}(x)=t(x) \cap H_{i}(i=$ $1,2)$, and set $V_{\xi}(x)=V_{t(x), K(x), \xi}(x)$ for every $\xi<2^{\mathfrak{c}}$. For every $C \in[X]^{\omega}$, let $\left\langle\xi_{j}(C)\right\rangle_{j<\omega}$ be a list, with repetitions permitted, of $t_{1}(C)=\bigcup_{x \in C} t_{1}(x)$. Since replacing $V(x)$ by a smaller neighborhood preserves $V(x) \subset G_{k}$, by induction on $\beta<\mathfrak{c}$ (and on $k \in \omega$ for each $\beta$ ) we can make sure that the following conditions also hold for every $\beta<\mathfrak{c}$ :

(4-1) if $\beta>\sup \pi\left(A_{\beta}\right)$, then $\left\{\xi_{j}\left(A_{\beta}\right): j<k\right\} \subset t_{1}(\beta, k)$ for every $k \in \omega$;

(4-2) $j<k<\omega$ implies $t_{1}(\beta, j) \subset t_{1}(\beta, k)$;

(4-3) each $V_{t(x), K(x)}(x)$ is a complete neighborhood of $x=\langle\beta, k\rangle$ for every $k \in \omega$.

For the rest of Sections 4 and 5 , we are going to assume that we have fixed a $V(x)=V_{t(x), K(x)}(x)$ satisfying (4-1), (4-2) and (4-3).

Next, let us fix two countable elementary submodels $M, N$ of $H\left(\left(2^{2^{c}}\right)^{+}\right)=\{$all sets whose transitive closure has cardinality $\left.\leq 2^{2^{\mathrm{c}}}\right\}$ in such a way that $M \in N$ and

$$
\mathfrak{c},\left\langle S_{\xi}\right\rangle_{\xi<2^{\mathfrak{c}}}, H_{1}, \quad t: X \rightarrow[H]^{<\omega}, \quad K: X \rightarrow[X]^{<\omega},\langle x(\xi)\rangle_{\langle\xi, x\rangle \in H_{1} \times X}
$$

are all elements of $M$.

Let $A=N \cap X(=(N \cap \mathfrak{c}) \times \omega))$ and $R=t_{1}(A) \cap M$. Note that by (4-1),

$$
\beta>\sup (N \cap \mathfrak{c}) \text { and } A_{\beta}=A \text { imply } R \subset \bigcup_{k \in \omega} t_{1}(\beta, k) \text {. }
$$

Definition 4.1. Let $\beta_{n}>\sup (N \cap \mathfrak{c}), n \in \omega$, be a sequence of ordinals. We say that a sequence $x_{n}=\left\langle\alpha_{n}, k_{n}\right\rangle \in N, n \in \omega$, is an increasing reflection of $\left\langle\beta_{n}\right\rangle_{n \in \omega}$ if the following conditions hold:

(4-5) for every $n \in \omega, t_{1}\left(x_{n}\right) \cap M=t_{1}\left(\beta_{n}, k_{n}\right) \cap M$, and whenever $\xi \in t_{1}\left(x_{n}\right) \cap M$, then $x_{n}(\xi)=\left\langle\beta_{n}, k_{n}\right\rangle(\xi)$; 
(4-6) $t_{1}\left(x_{n}\right) \backslash M, n \in \omega$, are pairwise disjoint;

(4-7) $k_{0}<\cdots<k_{n}<\cdots$, and for every $n \in \omega$ and $\xi \in \bigcup_{j<n} t_{2}\left(x_{j}\right), k_{n}>$ height of $S_{\xi}$

(4-8) if $A_{\beta_{n}}=A$ for every $n \in \omega$, then for every $\Theta \in R$ we have $\Theta \in t_{1}\left(x_{n}\right)$ for all but finitely many $n \in \omega$.

Lemma 4.2. For every sequence $\beta_{n}>\sup (N \cap \mathfrak{c}), n \in \omega$, of ordinals in $\mathfrak{c}$ there is an increasing reflection $\left\langle x_{n}\right\rangle_{n \in \omega}$ of $\left\langle\beta_{n}\right\rangle_{n \in \omega}$.

Proof. By induction on $n \in \omega$, we are going to define $\left\langle x_{n}\right\rangle_{n \in \omega}$ in such a way that (4-5), (4-6) and (4-7) are satisfied. ((4-8) will then automatically follow.)

Suppose $\left\langle x_{j}\right\rangle_{j<n}$ is defined. Then take $k_{n} \in \omega$ such that $k_{n}>k_{0}, \cdots, k_{n-1}$ and $\xi \in \bigcup_{j<n} t_{2}\left(x_{j}\right)$ implies $k_{n}>$ height of $S_{\xi}$. Define a finite function $r$ by setting $\operatorname{dom}(r)=t_{1}\left(\beta_{n}, k_{n}\right) \cap M$ and, for every $\xi \in \operatorname{dom}(r), r(\xi)=i$ iff $\left\langle\beta_{n}, k_{n}\right\rangle(\xi)=i$. Note that $r \in M$.

Consider the property

$\phi(\alpha): t_{1}\left(\alpha, k_{n}\right) \supset \operatorname{dom}(r)$ and $\left\langle\alpha, k_{n}\right\rangle(\xi)=r(\xi)$ for every $\xi \in \operatorname{dom}(r)$.

Note that $\phi(\alpha)$ can be described by a formula with all parameters from $M$, and that for $\beta_{n}>\sup (N \cap \mathfrak{c}), \phi\left(\beta_{n}\right)$ holds.

Let $D$ be a maximal subset of $\{\alpha \in \mathfrak{c}: \phi(\alpha)\}$ such that $t_{1}\left(\alpha, k_{n}\right) \backslash \operatorname{dom}(r), \alpha \in D$, are pairwise disjoint. Since $t_{1}, \mathfrak{c}, r \in M$, we can take such a $D \in M$. If $D$ was countable, then we would have $D \subset M$, and then $D \cup\left\{\beta_{n}\right\}$ would contradict the maximality of $D$. Hence $D$ is uncountable, and thus there is an $\alpha_{n} \in D$ such that

$$
\left(t_{1}\left(\alpha_{n}, k_{n}\right) \backslash \operatorname{dom}(r)\right) \cap\left(M \cup \bigcup_{j<n} t_{1}\left(x_{j}\right)\right)=\emptyset .
$$

Since $D, t_{1}, r, M,\left\langle x_{j}\right\rangle_{j<n} \in N$, we can pick such an $\alpha_{n} \in N$.

We are going to show that $x_{n}=\left\langle\alpha_{n}, k_{n}\right\rangle$ is as required to satisfy (4-5), (4-6) and $(4-7)$.

(4-5). Since $\phi\left(\alpha_{n}\right)$ holds, $t_{1}\left(x_{n}\right) \cap M \supset \operatorname{dom}(r)=t_{1}\left(\beta_{n}, k_{n}\right) \cap M$. By $(*)$

$$
\left(t_{1}\left(x_{n}\right) \backslash \operatorname{dom}(r)\right) \cap M=\emptyset,
$$

i.e.

$$
t_{1}\left(x_{n}\right) \cap M \subset \operatorname{dom}(r)=t_{1}\left(\beta_{n}, k_{n}\right) \cap M .
$$

Finally, for every $\xi \in \operatorname{dom}(r), x_{n}(\xi)=r(\xi)$ by $\phi\left(\alpha_{n}\right)$ and $\left\langle\beta_{n}, k_{n}\right\rangle(\xi)=r(\xi)$ by the definition of $r$; hence $x_{n}(\xi)=\left\langle\beta_{n}, k_{n}\right\rangle(\xi)$.

(4-6) By $(*), t_{1}\left(x_{n}\right) \backslash M=t_{1}\left(x_{n}\right) \backslash\left(t_{1}\left(x_{n}\right) \cap M\right)=t_{1}\left(x_{n}\right) \backslash \operatorname{dom}(r)$ is disjoint from $t\left(x_{j}\right) \backslash M$ for every $j<n$.

Finally, at the beginning of the proof of Lemma 4.2 , we chose $k_{n} \in \omega$ so that it satisfied (4-7).

Having constructed $\left\langle x_{n}\right\rangle_{n \in \omega}$ in $N$ satisfying (4-5) through (4-7), it only remains to prove that $\left\langle x_{n}\right\rangle_{n \in \omega}$ also satisfies (4-8). To prove this, suppose that $A_{\beta_{n}}=A$ for every $n \in \omega$, and let $\Theta \in R\left(=t_{1}(A) \cap M\right)$. Then $\Theta=\xi_{j}(A)$ for some $j<\omega$. We are going to show that $\Theta \in t_{1}\left(x_{n}\right)$ for every $n>j$. Fix $n$. Since $k_{n} \geq n$, it follows from $A_{\beta_{n}}=A$ and (4-1) that $\Theta \in t_{1}\left(\beta_{n}, k_{n}\right)$. Since $\Theta \in R \subset M$, we conclude that $\Theta \in t_{1}\left(\beta_{n}, k_{n}\right) \cap M=t_{1}\left(x_{n}\right) \cap M \subset t_{1}\left(x_{n}\right)$.

When defining control pairs $\langle A, d\rangle$ in Sections 5 and 6 , the following observation will be useful.

Proposition 4.3. If $\xi, \eta \in N \cap H$ and $S_{\xi} \uparrow A=S_{\eta} \uparrow A$, then $\xi=\eta$. 
Proof. Since $\left\langle S_{\xi}\right\rangle_{\xi<2^{\mathrm{c}}} \in M \subset N$, it follows that $\xi, \eta \in N$ and $S_{\xi}\left\lceil A=S_{\eta} \uparrow A\right.$ imply $N \models S_{\xi}=S_{\eta}$, which in turn implies $S_{\xi}=S_{\eta}$. Then $\xi=\eta$ by Proposition 1.2 .

\section{5. $X$ is not Countably paracompact: $\bigcap_{k \in \omega} G_{k} \neq \emptyset$}

Let $\beta, \gamma \in \mathfrak{c}$ and $\xi \in H_{1}$. We shall write $\beta \approx_{\xi} \gamma$ iff $\langle\beta, k\rangle(\xi)=\langle\gamma, k\rangle(\xi)$ for every $k \in \omega$. We will say that $\gamma$ is $\xi$-homogeneous iff either $\{\gamma\} \times \omega \subset B_{\xi}^{0}$ or $\{\gamma\} \times \omega \subset B_{\xi}^{1}$. Otherwise we call $\gamma \xi$-splitting. We shall say that $k \in \omega$ is above the split of $\xi$ at $\gamma$ iff both $(\{\gamma\} \times(k+1)) \cap B_{\xi}^{0} \neq \emptyset$ and $(\{\gamma\} \times(k+1)) \cap B_{\xi}^{1} \neq \emptyset . \gamma$ will be called R-homogeneous if $\gamma$ is $\xi$-homogeneous for every $\xi \in R$.

Lemma 5.1. There is a $\gamma>\sup (N \cap \mathfrak{c})$ which is $R$-homogeneous.

Proof. Suppose indirectly that there is no such $\gamma$. Then to get a contradiction, we are going to construct a strictly increasing $\omega_{1}$-sequence $\left\langle\Theta_{\nu}\right\rangle_{\nu<\omega_{1}}$ of ordinals in $R$, contradicting the countability of $R$.

For every $\gamma>\sup (N \cap \mathfrak{c})$, let $\Theta(\gamma)$ denote the smallest $\Theta \in R$ such that $\gamma$ is not $\Theta$-homogeneous.

A sequence $\left\langle\gamma_{\nu}\right\rangle_{\nu<\omega_{1}}$ will be called a consistent $\omega_{1}$-sequence iff the following conditions hold for every $\nu<\omega_{1}$ :

(5-1) if $\mu<\nu$, then $\sup (N \cap \mathfrak{c})<\gamma_{\mu}<\gamma_{\nu}$;

(5-2) with the notation $\Theta\left(\gamma_{\nu}\right)=\Theta_{\nu}, \mu<\nu$ implies $\Theta_{\mu}<\Theta_{\nu}$;

(5-3) $A_{\gamma_{\nu}}=A$;

(5-4) if $\mu<\nu$ and $\xi \in R \cap \Theta_{\mu}$, then $\gamma_{\mu} \approx_{\xi} \gamma_{\nu}$.

We are going to build a consistent $\omega_{1}$-sequence $\left\langle\gamma_{\nu}\right\rangle_{\nu<\omega_{1}}$ by transfinite induction. To start, take any $\gamma_{0}>\sup (N \cap \mathfrak{c})$ such that $A_{\gamma_{0}}=A$. Then (5-1) through (5-4) are clearly satisfied for $\nu=0$.

Suppose now that $0<\delta<\omega_{1}$ and for every $\nu<\delta$ we have constructed $\gamma_{\nu}$ in such a way that (5-1) through (5-4) are satisfied. Then we split finding $\gamma_{\delta}$ into two cases.

Case 1. Suppose $\delta=\nu+1$ is a successor ordinal. Then by Lemma 4.2 we can take an increasing reflection $x_{n}=\left\langle\alpha_{n}, k_{n}\right\rangle \in N, n \in \omega$, of the constant sequence $\beta_{n}=\gamma_{\nu}$. Fix $i \in\{0,1\}$ such that $x_{n}\left(\Theta_{\nu}\right)=i$ for infinitely many $n \in \omega$. Then let us define the function $d$ by setting $\operatorname{dom}(d)=\left\{S_{\xi}\left\lceil A: \xi \in \bigcup_{n \in \omega} t\left(x_{n}\right) \cap \Theta_{\nu} \cup\left\{\Theta_{\nu}\right\}\right\}\right.$, and, with the notation $t^{*}\left(x_{n}\right)=t\left(x_{n}\right) \cap \Theta_{\nu}$, by letting

$$
d\left(S_{\xi}\lceil A)=\left\{\begin{array}{l}
x_{n}(\xi), \text { if } \xi \in t^{*}\left(x_{n}\right) \backslash \bigcup_{j<n} t^{*}\left(x_{j}\right) ; \\
i \text { if } \xi=\Theta_{\nu} .
\end{array}\right.\right.
$$

Note that $\bigcup_{n \in \omega} t\left(x_{n}\right) \cap \Theta_{\nu} \cup\left\{\Theta_{\nu}\right\} \subset N \cap H$, and thus, by Proposition 4.3, $d$ is well-defined.

Now let $\beta \in \mathfrak{c}$ be such that $\beta>\gamma_{\nu}$ and $\left\langle A_{\beta}, d_{\beta}\right\rangle=\langle A, d\rangle$, and set $\Theta=\Theta(\beta)$. We are going to show that choosing $\gamma_{\delta}=\beta$ satisfies (5-1) through (5-4) (with $\delta$ in place of $\nu)$.

$(5-1)$ and (5-3) follow from the definition of $\beta$.

To prove (5-2) it is enough to prove that $\Theta>\Theta_{\nu}$. Let $y=\langle\beta, 0\rangle$, and for every $n \in \omega$, let $y[n]=\{\beta\} \times\left(k_{n}+1\right)$. Fix $n \in \omega$. We will show that $y[n] \subset$ $V_{\Theta_{\nu}}\left(x_{n}\right)\left(=V_{t\left(x_{n}\right), K\left(x_{n}\right), \Theta_{\nu}}\left(x_{n}\right)=\bigcap_{\xi \in t\left(x_{n}\right) \cap \Theta_{\nu}} T_{\xi}\left(x_{n}\right) \cap\left(W_{k_{n}+1} \backslash K\left(x_{n}\right)\right)\right.$; cf. the beginning of Section 4 and the beginning of Section 3$)$. 
Since $K\left(x_{n}\right) \subset N$ and $\beta \notin N, y[n] \subset W_{k_{n+1}} \backslash K\left(x_{n}\right)$. By induction on $\xi \in$ $t\left(x_{n}\right) \cap \Theta_{\nu}=t^{*}\left(x_{n}\right)$, we are going to show that $\xi \in t^{*}\left(x_{n}\right)$ implies

$$
\left(I_{\xi}\right) y[n] \subset T_{\xi}\left(x_{n}\right) \text {. }
$$

Suppose $\xi \in t^{*}\left(x_{n}\right)$ and that for every $\eta \in t^{*}\left(x_{n}\right) \cap \xi,\left(I_{\eta}\right)$ holds. Then $y[n] \subset$ $V_{\xi}\left(x_{n}\right) \subset S_{\xi}^{x_{n}(\xi)}$. Thus by Observation 1.3, to prove $\left(I_{\xi}\right)$ it is enough to show that

$$
d\left(S_{\xi} \uparrow A\right)=x_{n}(\xi)
$$

To prove $(*)$, recall that $\xi \in t^{*}\left(x_{n}\right)$. If $\xi \notin \bigcup_{j<n} t^{*}\left(x_{j}\right)$, then $(*)$ follows directly from the definition of $d$.

So suppose that there is a $j<n$ such that $\xi \in t^{*}\left(x_{j}\right) \backslash \bigcup_{\ell<j} t^{*}\left(x_{\ell}\right)$. Then

$$
d\left(S_{\xi} \uparrow A\right)=x_{j}(\xi)
$$

by the definition of $d$.

If $\xi \in H_{2}$, then our assumptions that $\xi \in t^{*}\left(x_{n}\right)$ and $\xi \in t^{*}\left(x_{j}\right)$ contradict (4-7) in the definition of an increasing reflection. Similarly, $\xi \in H_{1} \backslash M$ contradicts (4-6). Thus only $\xi \in H_{1} \cap M$ is possible. Then $\xi \in t_{1}\left(x_{j}\right) \cap M$, so by (4-5), $x_{j}(\xi)=\left\langle\gamma_{\nu}, k_{j}\right\rangle(\xi)$. (Recall that $\beta_{j}=\gamma_{\nu}$ for every $j \in \omega$.) Now note that $\xi \in$ $t^{*}\left(x_{j}\right) \cap H_{1} \cap M=t\left(x_{j}\right) \cap \Theta_{\nu} \cap H_{1} \cap M=t_{1}\left(x_{j}\right) \cap M \cap \Theta_{\nu} \subset R \cap \Theta_{\nu}$, and thus $\gamma_{\nu}$ is $\xi$-homogeneous. Hence $\left\langle\gamma_{\nu}, k_{j}\right\rangle(\xi)=\left\langle\gamma_{\nu}, k_{n}\right\rangle(\xi)$. Since $\xi \in t_{1}\left(x_{n}\right) \cap M$, by (4-5), $\left\langle\gamma_{\nu}, k_{n}\right\rangle(\xi)=x_{n}(\xi)$. We conclude that $x_{j}(\xi)=x_{n}(\xi)$ and hence $(*)$ holds.

Having proved $\left(I_{\xi}\right)$ for every $\xi \in t^{*}\left(x_{n}\right)$ we conclude that

$$
y[n] \subset V_{\Theta_{\nu}}\left(x_{n}\right) \text { for every } n \in \omega .
$$

We can now finish the proof of $\Theta>\Theta_{\nu}$ in the following steps.

1. $\Theta<\Theta_{\nu}$ is impossible. Indeed, if $\Theta<\Theta_{\nu}$, then by $\Theta \in R$ and by (4-8) we can pick an $n \in \omega$ such that $\Theta \in t_{1}\left(x_{n}\right)$ and $k_{n}$ is above the split of $\Theta$ at $\beta$. Since $\Theta \in t^{*}\left(x_{n}\right)=t\left(x_{n}\right) \cap \Theta_{\nu}$, we conclude by $\left(I_{\Theta}\right)$ that $y[n] \subset T_{\Theta}\left(x_{n}\right)$, in contradiction with our assumption that $k_{n}$ is above the split of $\Theta$ at $\beta$.

2. $\Theta=\Theta_{\nu}$ is impossible. To see this, pick an $n \in \omega$ such that $x_{n}\left(\Theta_{\nu}\right)=i$ and $k_{n}$ is above the split of $\Theta$ at $\beta$. We have already proved that $y[n] \subset$ $V_{\Theta_{\nu}}\left(x_{n}\right) \subset S_{\Theta_{\nu}}^{x_{n}\left(\Theta_{\nu}\right)}=S_{\Theta_{\nu}}^{i}$. By the definition of $d, d\left(S_{\Theta_{\nu}} \uparrow A\right)=i$. Since $\left\langle A_{\beta}, d_{\beta}\right\rangle=\langle A, d\rangle$, by Observation 1.3, it follows that $y[n] \subset B_{\Theta_{\nu}}^{i}$. Then $\Theta=\Theta_{\nu}$ would contradict our assumption that $k_{n}$ is above the split of $\Theta$ at $\beta$.

It only remains to prove that (5-4) holds for $\gamma_{\delta}=\beta$, i.e. $\mu<\delta$ and $\xi \in R \cap \Theta_{\mu}$ imply $\gamma_{\mu} \approx_{\xi} \beta$. Since $\delta=\nu+1$ and (5-4) holds for $\nu$, it is enough to show that $\xi \in R \cap \Theta_{\nu}$ implies $\gamma_{\nu} \approx_{\xi} \beta$. Since $\xi<\Theta_{\nu}<\Theta$, both $\gamma_{\nu}$ and $\beta$ are $\xi$-homogeneous. Thus it suffices to find at least one $k_{n} \in \omega$ such that $\left\langle\gamma_{\nu}, k_{n}\right\rangle(\xi)=\left\langle\beta, k_{n}\right\rangle(\xi)$. To do this, let $n \in \omega$ be so big that $\xi \in t_{1}\left(x_{n}\right)$. (Such an $n$ exists by (4-8).) Then $\xi \in t\left(x_{n}\right) \cap \Theta_{\nu}=t^{*}\left(x_{n}\right)$; hence by $\left(I_{\xi}\right), y[n] \subset T_{\xi}\left(x_{n}\right)$. In particular, $\left\langle\beta, k_{n}\right\rangle(\xi)=x_{n}(\xi)$. On the other hand, $\xi \in t_{1}\left(x_{n}\right) \cap R=t_{1}\left(x_{n}\right) \cap M$. Hence by $(4-5), x_{n}(\xi)=\left\langle\gamma_{\nu}, k_{n}\right\rangle(\xi)$. Hence $\left\langle\beta, k_{n}\right\rangle(\xi)=x_{n}(\xi)=\left\langle\gamma_{\nu}, k_{n}\right\rangle(\xi)$.

Case 2. Suppose that $\delta<\omega_{1}$ is a limit ordinal. Let us pick a strictly increasing sequence $\left\langle\delta_{n}\right\rangle_{n \in \omega}$ of ordinals cofinal in $\delta$, and let's set $\beta_{n}=\gamma_{\delta_{n}}$ for every $n \in \omega$. Let $\left\langle x_{n}\right\rangle_{n \in \omega}, x_{n}=\left\langle\alpha_{n}, k_{n}\right\rangle$, be an increasing reflection of $\left\langle\beta_{n}\right\rangle_{n \in \omega}$. Define the function 
$d$ by setting $\operatorname{dom}(d)=\left\{S_{\xi} \mid A: \xi \in \bigcup_{n \in \omega} t\left(x_{n}\right) \cap \Theta_{\delta_{n}}\right\}$, and, with the notation $t^{*}\left(x_{n}\right)=t\left(x_{n}\right) \cap \Theta_{\delta_{n}}\left(=t\left(x_{n}\right) \cap \Theta\left(\gamma_{\delta_{n}}\right)\right)$, by letting

$$
d\left(S_{\xi}\lceil A)=x_{n}(\xi) \text { for every } \xi \in t^{*}\left(x_{n}\right) \backslash \bigcup_{j<n} t^{*}\left(x_{j}\right) .\right.
$$

Since $\bigcup_{n \in \omega} t^{*}\left(x_{n}\right) \subset N \cap H, d$ is well-defined by Proposition 4.3. Now let $\beta \in \mathfrak{c}$ be such that $\beta>\sup \left\{\gamma_{\delta_{n}}: n \in \omega\right\}$ and $\left\langle A_{\beta}, d_{\beta}\right\rangle=\langle A, d\rangle$, and let $\Theta=\Theta(\beta)$. We are going to show that choosing $\gamma_{\delta}=\beta$ satisfies (5-1) through (5-4) (with $\delta$ in place of $\nu)$.

(5-1) and (5-3) follow from the definition of $\beta$.

In order to start the proof of (5-2), let $y=\langle\beta, 0\rangle$, and for every $n \in \omega$, let $y[n]=\{\beta\} \times\left(k_{n}+1\right)$.

Fix $n \in \omega$. We will show that $y[n] \subset V_{\Theta_{\delta_{n}}}\left(x_{n}\right)$. Since $K\left(x_{n}\right) \subset N$ and $\beta \notin$ $N, y[n] \subset W_{k_{n}+1} \backslash K\left(x_{n}\right)$. By induction on $\xi \in t^{*}\left(x_{n}\right)$ we are going to show that $\xi \in t^{*}\left(x_{n}\right)$ implies

$$
y[n] \subset T_{\xi}\left(x_{n}\right) .
$$

Suppose that $\xi \in t^{*}\left(x_{n}\right)$ and that for every $\eta \in t^{*}\left(x_{n}\right) \cap \xi,\left(J_{\eta}\right)$ holds. Then $y[n] \subset V_{\xi}\left(x_{n}\right) \subset S_{\xi}^{x_{n}(\xi)}$. Thus by Observation 1.3 , to prove $\left(J_{\xi}\right)$ it is enough to show that

$$
d\left(S_{\xi}\lceil A)=x_{n}(\xi)\right.
$$

To prove $(* *)$ recall that $\xi \in t^{*}\left(x_{n}\right)$. If $\xi \notin \bigcup_{j<n} t_{j}^{*}\left(x_{j}\right)$, then $(* *)$ follows directly from the definition of $d$.

So suppose that there is a $j<n$ such that $\xi \in t^{*}\left(x_{j}\right) \backslash \bigcup_{\ell<j} t^{*}\left(x_{\ell}\right)$. Then

$$
d\left(S_{\xi} \uparrow A\right)=x_{j}(\xi)
$$

by the definition of $d$. Since $\xi \in H_{2}$ would contradict (4-7) and $\xi \in H_{1} \backslash M$ would contradict (4-6), it follows that $\xi \in H_{1} \cap M$. Then $\xi \in t_{1}\left(x_{j}\right) \cap M$, so by (4-5), $x_{j}(\xi)=\left\langle\gamma_{\delta_{j}}, k_{j}\right\rangle(\xi)$. Since $\xi \in t^{*}\left(x_{j}\right) \subset R \cap \Theta_{\delta_{j}}$ and $\delta_{j}<\delta_{n}$, it follows that $\gamma_{\delta_{j}} \approx_{\xi} \gamma_{\delta_{n}}$. Hence $\left\langle\gamma_{\delta_{j}}, k_{j}\right\rangle(\xi)=\left\langle\gamma_{\delta_{n}}, k_{j}\right\rangle(\xi)$. Since $\xi<\Theta_{\delta_{j}}<\Theta_{\delta_{n}}, \gamma_{\delta_{n}}$ is $\xi$ homogeneous. Hence $\left\langle\gamma_{\delta_{n}}, k_{j}\right\rangle(\xi)=\left\langle\gamma_{\delta_{n}}, k_{n}\right\rangle(\xi)$. Finally $\xi \in t_{1}\left(x_{n}\right) \cap M$, so by $(4-5),\left\langle\gamma_{\delta_{n}}, k_{n}\right\rangle(\xi)=x_{n}(\xi)$. The sequence of equalities just proved shows that $d\left(S_{\xi}\lceil A)=x_{j}(\xi)=x_{n}(\xi)\right.$.

Having proved $\left(J_{\xi}\right)$ for every $\xi \in t^{*}\left(x_{n}\right)$ and $n \in \omega$, we can easily finish the proof of $\Theta \geq \sup \left\{\Theta_{\delta_{n}}: n \in \omega\right\}$. Let us assume indirectly that $\Theta<\Theta_{\delta_{n}}$ for some $n \in \omega$. By (4-8) we can assume that $\Theta \in t_{1}\left(x_{n}\right)$ and $k_{n}$ is above the split of $\Theta$ at $\beta$. Since $\Theta \in t^{*}\left(x_{n}\right)=t\left(x_{n}\right) \cap \Theta_{\delta_{n}}$, we conclude by $\left(J_{\Theta}\right)$ that $y[n] \subset T_{\Theta}\left(x_{n}\right)$, in contradiction with our assumption that $k_{n}$ is above the split of $\Theta$ at $\beta$.

Finally, to prove that (5-4) holds for $\gamma_{\delta}=\beta$, it is enough to verify that for every $n \in \omega$ and $\xi \in R \cap \Theta_{\delta_{n}}, \gamma_{\delta_{n}} \approx_{\xi} \beta$. Fix $n \in \omega$ and $\xi \in R \cap \Theta_{\delta_{n}}$. Since $\xi<\Theta_{\delta_{n}}<\Theta$, both $\gamma_{\delta_{n}}$ and $\beta$ are $\xi$-homogeneous. Thus it suffices to find at least one $k_{n} \in \omega$ such that $\left\langle\gamma_{\delta_{n}}, k_{n}\right\rangle(\xi)=\left\langle\beta, k_{n}\right\rangle(\xi)$. To do this, pick, by (4-8), an $n$ such that $\xi \in t_{1}\left(x_{n}\right)$. Then $\xi \in t\left(x_{n}\right) \cap \Theta_{\delta_{n}}=t^{*}\left(x_{n}\right)$, so by $\left(J_{\xi}\right), y[n] \subset T_{\xi}\left(x_{n}\right)$. In particular, $\left\langle\beta, k_{n}\right\rangle(\xi)=x_{n}(\xi)$. On the other hand $\xi \in t_{1}\left(x_{n}\right) \cap R=t_{1}\left(x_{n}\right) \cap M$, so by $(4-5), x_{n}(\xi)=\left\langle\gamma_{\delta_{n}}, k_{n}\right\rangle(\xi)$. Hence $\left\langle\beta, k_{n}\right\rangle(\xi)=\left\langle\gamma_{\delta_{n}}, k_{n}\right\rangle(\xi)$.

We have completed the construction of a consistent $\omega_{1}$-sequence and thus the proof of Lemma 5.1 . 
The following corollary finishes the proof that $X$ is not countably paracompact.

Corollary 5.2. $\bigcap_{k \in \omega} G_{k} \neq \emptyset$.

Proof. Note first that by $B_{\xi_{k}}^{1} \subset G_{k}$, it is enough to prove that $\bigcap_{k \in \omega} B_{\xi_{k}}^{1} \neq \emptyset$. Also note that since $B_{\xi_{k}}^{0} \subset W_{k}$, a $\xi_{k}$-homogeneous $\gamma<\mathfrak{c}$ has the property that $\{\gamma\} \times \omega \subset B_{\xi_{k}}^{1}$. Therefore $\bigcap_{k \in \omega} B_{\xi_{k}}^{1} \neq \emptyset$ will follow from Lemma 5.1, if we can show that $\left\{\xi_{k}: k \in \omega\right\} \subset R$. To see this, let $\alpha \in M \cap \mathfrak{c}$. Then by our choice of $V_{t(x), K(x)}(x)$ at the beginning of Section $4,\left\{\xi_{k}: k \in \omega\right\} \subset \bigcup_{k \in \omega} t_{1}(\alpha, k) \subset t_{1}(A) \cap M=R$.

\section{Concluding Remarks}

1. F. Tall ([T1], $[\mathrm{T} 2])$ asked whether normal, screenable spaces have to be collectionwise normal. We can now answer Tall's question with the help of the following result of M.E. Rudin.

Theorem 6.1 (M.E. Rudin [R2]). If there is a normal, screenable, nonparacompact space, then there is a normal, screenable space which is not collectionwise normal.

Thus our Theorem I implies

Theorem 6.2. $\quad$ There is a normal, screenable, space which is not collectionwise normal.

2. Several related problems remain open. In particular:

Problem 6.3. Is there a nonparacompact normal space with a $\sigma$-disjoint base?

Problem 6.4. Is there a normal, screenable, nonparacompact space which is first countable?

\section{REFERENCES}

[B] R.H. Bing, Metrization of Topological Spaces, Canad. J. Math. 3 (1951), 175-186. MR 13:264f

[D] C.H. Dowker, On Countably Paracompact Spaces, Canad. J. Math. 3 (1951), 219-224. MR 13:264c

[KV] K. Kunen and J.E. Vaughan, eds., Handbook of Set-theoretic Topology, North-Holland, 1984. MR 85k:54001

[N] K. Nagami, Paracompactness and strong screenability, Nagoya Math. J. 8 (1955), 83-88. MR 16:1138d

[R1] M.E. Rudin, A Normal, Screenable, Non-paracompact Space, Topology Appl. 15 (1983), 313-322. MR 84d:54042

[R2] M.E. Rudin, Collectionwise Normality in Screenable Spaces, PAMS 87 (1983), 347-350. MR 84a:54038

[T1] F.D. Tall, Normality versus Collectionwise Normality, Handbook of Set-theoretic Topology (K. Kunen and J. Vaughan, eds.), North-Holland, 1984, pp. 685-732. MR 86m:54022

[T2] F.D. Tall, Tall's Problems, Open Problems in Topology (J. van Mill, G.M. Reed, eds.), North-Holland, 1990, pp. 21-35. MR 92c:54001

[TP] Topology Proceedings, vol. 1, 1976, pp. 363-364, Problem Section.

[W] S. Watson, Problems I wish I could solve, Open Problems in Topology (J. van Mill and G.M. Reed, eds.), North-Holland, 1990, pp. 37-76. MR 92c:54001

Department of Mathematics and Statistics, Miami University, Oxford, Ohio 45058

E-mail address: ZTBalogh@miavx1.muohio.edu 